\title{
Relationship between the TaqI B polymorphism of the cholesterol ester transfer protein gene and atrial fibrillation in Han and Kazak populations
}

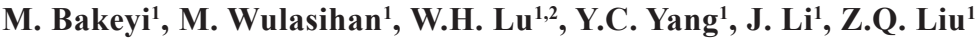 \\ and P.Y. $\mathrm{He}^{1}$ \\ ${ }^{1}$ Department of Cardiology, First Affiliated Hospital of Xinjiang Medical University, \\ Urumqi, China \\ ${ }^{2}$ Research Institution of Cardiology, \\ First Affiliated Hospital of Xinjiang Medical University, Urumqi, China \\ Corresponding author: M. Wulasihan \\ E-mail: muhuyati@163.com
}

Genet. Mol. Res. 13 (4): 9300-9307 (2014)

Received May 21, 2013

Accepted December 19, 2013

Published February 14, 2014

DOI http://dx.doi.org/10.4238/2014.February.14.4

\begin{abstract}
The TaqI B polymorphism in the cholesterol ester transfer protein (CETP) (B1 and B2 alleles; rs708272) is associated with changes in enzyme activity and lipid concentrations. The B1 allele of the CETP gene is a known independent risk factor for genetic susceptibility to atrial fibrillation (AF); however, little is known about this polymorphism in the minority groups of Xinjiang, China. We examined the role of this polymorphism in AF using two independent case-control studies: the Han population (101 AF patients and 129 control subjects) and the Kazak population (103 AF patients and 101 control subjects). Carriers of the B1B1 genotype were more frequent among AF patients than among controls both in the Han population (34.7 versus $26.4 \% ; \chi^{2}=10.686, \mathrm{P}$ $=0.001)$ and in the Kazak population (53.4 versus $24.8 \% ; \chi^{2}=27.802$, $\mathrm{P}<0.001)$. The odds ratio $(\mathrm{OR})$ for carriers of the B1B1 genotype to
\end{abstract}


AF susceptibility was 0.187 [95\% confidence interval $(\mathrm{CI})=0.071$ $0.491]$ in the Han group and $8.426(95 \% \mathrm{CI}=2.295-30.933)$ in the Kazak population. After adjustment of confounding factors such as gender, age, smoking, alcohol consumption, hypertension, diabetes, as well as serum levels of triglyceride, total cholesterol, and high-density lipoprotein, the difference remained significant in the Han group $(\mathrm{P}=0.001 ; \mathrm{OR}=0.187$, $95 \% \mathrm{CI}=0.071-0.491)$ and in the Kazak group $(\mathrm{P}=0.001 ; \mathrm{OR}=8.426$, $95 \% \mathrm{CI}=2.295-30.933)$. The presence of the $\mathrm{B} 1 \mathrm{~B} 1$ polymorphism of the Taq1B CETP genotype contributes to the development of AF in the Han and Kazak populations in western China (Xinjiang).

Key words: Atrial fibrillation; CETP; Polymorphism; Polymerase chain reaction-restriction fragment length polymorphism

\section{INTRODUCTION}

Atrial fibrillation (AF) is one of the most common types of arrhythmia. Because of its serious complications, AF has received universal attention. Epidemiological studies have shown that the incidence of AF is $0.9 \%$ in the United States and $0.77 \%$ in China, whereas the incidence in Kazak adults of Xinjiang, China, is only 0.37\% (Nattel, 2002; Zhou et al., 2004; Lu et al., 2012). The incidence of AF in the Kazak population is obviously lower than that of other populations, and their clinical features also differ from those of the Han Chinese population. The molecular basis for the development of structural remodeling of fibrillating human atria is still controversial and remains under investigation. Some researchers have explained its genetic mechanisms, finding that some ion channels, inflammation, angiotensin-converting enzyme (ACE), and cholesterol ester transfer protein (CETP) genetic polymorphisms are associated with AF in different populations. However, there have been no studies on CETP polymorphisms in the ethnic minorities of Xinjiang. Albers, in 1984, first isolated CETP from serum. CETP is located in the long arm of chromosome 16 (16q $12-21$ ), is approximately $25 \mathrm{~kb}$ in length, is close to the lecithin cholesterol acyl transferase gene locus, and contains 16 exons and 15 introns. CETP is highly polymorphic. Clinical studies have demonstrated a robust association of the TaqI B allele in intron 1 (rs708272 C.T.; TaqI B minor allele frequency $=0.44$ ) with low CETP activity, decreased total cholesterol, and increased high-density lipoprotein cholesterol (HDL-C) levels. CETP is a hydrophobic glycoprotein consisting of 476 amino acids. It is an important lipid transfer protein in lipid metabolism, and mainly induces the reversal transport of excessive cholesterol from peripheral tissues and cells to liver metabolism through HDL, and accelerates the flow of cholesterol in peripheral tissues, maintaining the balance of cellular cholesterol levels.

CETP exchanges triglycerides (TG) from very low-density lipoprotein and low-density lipoprotein (LDL) particles for cholesterol esters from HDL, and selectively enhances liver HDL cholesterol ester uptake (Gauthier et al., 2005). The reduction of HDL-C levels is an independent risk factor for cardiovascular diseases, and the expression of CETP plays a decisive role in lipid transferring activation and in the formation of lipoprotein.

Our aim of the present study was to assess the effects of the TaqI B polymorphism of CETP with the risk of AF. 


\section{MATERIAL AND METHODS}

\section{Ethical approval of the study protocol}

In this study, blood samples were obtained from all enrolled participants. This study was conducted in accordance with the Declaration of Helsinki, and the study was approved by the Ethics Committee of the First Affiliated Hospital of Xinjiang Medical University. Individual written consent forms were obtained from all participants, which is usually performed due to quality management issues in our hospital.

\section{Study subjects}

Two patient populations (Han and Kazak) with AF were studied independently. A total of 101 Han patients and 103 Kazak patients diagnosed with AF at the First Affiliated Hospital of Xinjiang Medical University from January 2010 to December 2012 were recruited for this study. Furthermore, 54 Kazak AF subjects were selected from the Epidemiological Survey of Atrial Fibrillation of Kazaks in Xinjiang (Mu et al., 2010; Lu et al., 2012). This was a large epidemiological investigation that evaluated the morbidity of AF in 30,000 Kazak subjects between October 2008 and December 2011, establishing the first database of AF for the Kazak population in Xinjiang, in the western region of China. AF is generally confirmed following the diagnosis of an episode as AF on more than two occasions $>6$ months apart by serial 12-lead electrocardiography (ECG) or a 24-h Holter monitoring. The diagnosis points based on ECG are as follows: $\mathrm{P}$ wave disappearance and substitution of irregular baseline fluctuation ( $\mathrm{F}$ wave) with a frequency of 350 to 600 per minute, and an extremely irregular ventricle rate (Li et al., 2012). Patients with AF were all above 18 years of age. The exclusion criteria were as follows: age $>75$ years, valvular heart disease, congenital heart disease, hyperthyroidism, malignancy, acute inflammatory disease within 2 weeks, and interracial marriage family history (Liu et al., 2012).

The control population comprised 129 Han and 101 Kazak healthy subjects, who were matched for ethnicity, age, and gender, recruited from the First Affiliated Hospital of Xinjiang Medical University from January 2010 to December 2012. These individuals did not have a history of AF or ECG signs of AF. Systemic arterial hypertension was defined as a systolic blood pressure $>140 \mathrm{mmHg}$ and/or a diastolic blood pressure $>90 \mathrm{mmHg}$ (Guidelines Subcommittee, 1999) on at least 2 separate occasions, or receiving anti-hypertensive treatment. Individuals who had consumed alcohol in the previous 6 months were considered alcohol drinkers. Individuals reporting regular smoking in the previous 6 months were considered current smokers (Xiang et al., 2009). Diabetes mellitus was defined as the presence of active treatment with insulin or an oral antidiabetic agent. For patients on dietary treatment, documentation of an abnormal fasting blood glucose level or glucose tolerance test based on the World Health Organization criteria was required for establishing a diabetes diagnosis (World Health Organization Study Group, 1985).

\section{Biochemical analysis}

Serum concentrations of total cholesterol (TC), TG, glucose, HDL-C, and LDL-C were measured using standard methods in the Clinical Laboratory Department of the First Affiliated Hospital of Xinjiang Medical University. 


\section{DNA extraction}

Venous blood was collected after an overnight fast. For DNA extraction, whole venous blood was collected in tubes containing $0.17 \mathrm{M}$ ethylenediaminetetracetic acid (EDTA), and immediately stored at $-20^{\circ} \mathrm{C}$. Genomic DNA was extracted from peripheral vein blood leukocytes using a whole blood genome extraction kit (TIANGEN Biotech Corporation, Beijing, China).

\section{Primer design and CETP gene sequencing}

The primers were designed using the Primer Premier 5.0 software. Briefly, genotyping for the TaqI B polymorphism of the CETP gene was confirmed using polymerase chain reaction (PCR)-restriction fragment length polymorphism analysis.

The forward primer was 5'-CACTAGCCCAGAGAGAGGAGTGCC-3' and the reverse primer was 5'-CTGAGCCCAGCCGCACACTAA-3'.

The annealing temperature was $63^{\circ} \mathrm{C}$. Ten microliters of PCR product was incubated overnight with $1 \mu \mathrm{L} \mathrm{TaqI}$ in a total volume of $20 \mu \mathrm{L}$ at $37^{\circ} \mathrm{C}$, and the resulting fragments were separated on 3.0\% agarose gel. Absence of the TaqI B variant created a TaqI site that produced two fragments of 174 and $354 \mathrm{bp}$, respectively (Figure 1). Sequencing reactions were carried out by BGI (Beijing, China).

\section{A}
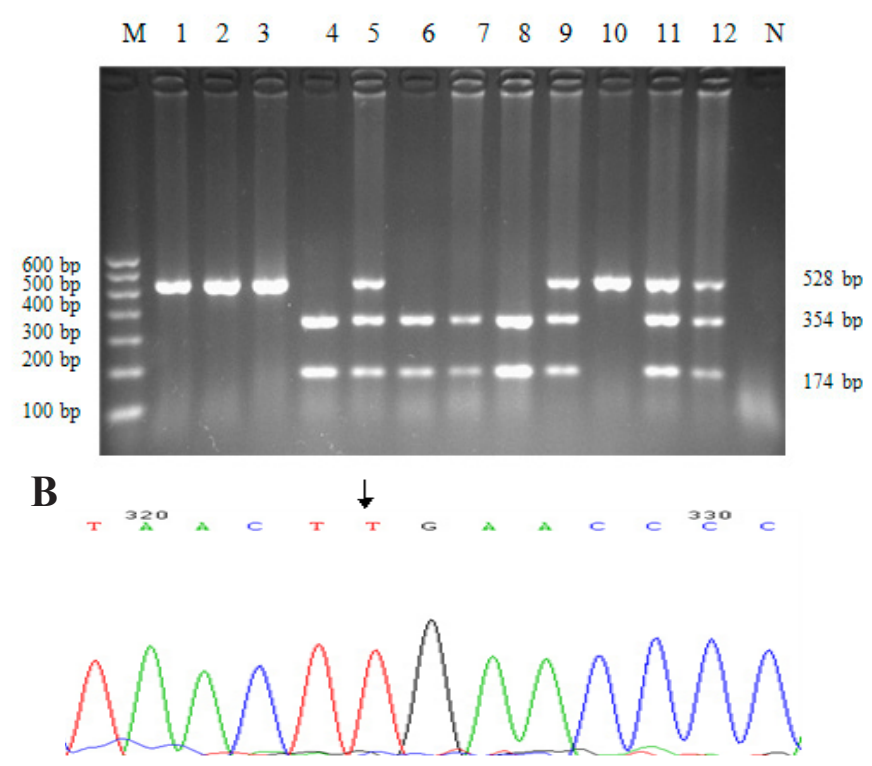

Figure 1. Restriction fragment length polymorphism analyses for determination of genotype A. B1B2 genotype showing three bands of 528, 354, and 174 bp (lanes 5, 9, 11, and 12); B1B1 genotype showing two bands of 354 and 174 bp (lanes 4, 6, 7 and 8); B2B2 genotype showing one band of 528 bp (lanes 1, 2, 3, and 10). $M$ is the marker of 100 to $700 \mathrm{bp}$; lane $N$ is the primer blank control group. B. Nucleotide sequences of the TaqI polymorphism.

\section{Statistical analyses}

Statistical analyses were conducted using the SPSS 17.0 software. Continuous data 
are reported as means $\pm \mathrm{SD}$, and the differences between $\mathrm{AF}$ patients and control subjects were assessed using the two independent-sample Student $t$-test. The Hardy-Weinberg equilibrium and differences in enumeration data between AF patients and control subjects were analyzed using the chi-squared test. The allelic frequency distribution was analyzed using $2 \times 2$-contingency tables at a $5 \%$ significance level. Logistic regression analyses were used to assess the contribution of major risk factors.

\section{RESULTS}

\section{Characteristics of study participants} in Table 1.

The clinical and laboratory characteristics of patients and control subjects are reported

\begin{tabular}{|c|c|c|c|c|c|c|c|c|}
\hline & \multicolumn{4}{|c|}{ Han } & \multicolumn{4}{|c|}{ Kazak } \\
\hline & Control $(\mathrm{N}=129)$ & $\mathrm{AF}(\mathrm{N}=101)$ & $\chi^{2}$ or $t$ & $P$ value & Control $(\mathrm{N}=101)$ & $\mathrm{AF}(\mathrm{N}=103)$ & $\chi^{2}$ or $t$ & $P$ value \\
\hline Age [mean (SD)] & $58.87(15.34)$ & $61.12(14.45)$ & 1.133 & 0.731 & $52.73(11.92)$ & $52.56(12.75)$ & -0.676 & 0.397 \\
\hline Male/female (\%) & $77 / 52$ & $68 / 33$ & 1.418 & 0.234 & $66 / 35$ & $68 / 35$ & 0.010 & 0.919 \\
\hline Hypertension [N (\%)] & $75(58.14)$ & $40(39.60)$ & 7.785 & 0.005 & $71(70.29)$ & $54(52.42)$ & 6.863 & 0.09 \\
\hline Drinking, $[\mathrm{N}(\%)]$ & $27(20.93)$ & $22(21.78)$ & 0.025 & 0.876 & $29(28.71)$ & $17(16.5)$ & 4.352 & 0.037 \\
\hline Smoking, [N (\%)] & $25(19.38)$ & $29(28.71)$ & 2.746 & 0.097 & $33(32.67)$ & 35 (33.98) & 0.039 & 0.843 \\
\hline BMI [mean (SD)] & $25.05(4.70)$ & $24.49(5.35)$ & -0.838 & 0.845 & $28.90(6.12)$ & $26.35(4.83)$ & -3.42 & 0.077 \\
\hline SBP [mean (SD)] & $131.25(21.13)$ & $123.18(22.95)$ & -2.764 & 0.200 & $127.82(16.59)$ & $140.30(21.67)$ & 4.612 & 4.866 \\
\hline DBP [mean (SD)] & $80.82(13.87)$ & $76.56(12.60)$ & -2.404 & 0.770 & $77.82(10.11)$ & $85.66(11.73)$ & 5.108 & 0.008 \\
\hline FBG $[$ mean $(\mathrm{SD})]$ & $5.74(0.87)$ & $6.41(6.51)$ & 1.609 & 0.003 & $4.95(0.69)$ & $6.09(6,26)$ & 1.825 & 0.020 \\
\hline TG [mean (SD)] & $3.23(8.54)$ & $1.38(0.99)$ & -2.156 & 0.004 & $1.61(1.30)$ & $1.13(0.71)$ & -3.32 & 0.001 \\
\hline TC [mean (SD)] & $4.50(1.13)$ & $4.14(1.03)$ & -2.526 & 0.313 & $4.54(1.10)$ & $4.56(1.08)$ & 0.082 & 0.859 \\
\hline HDL-C [mean (SD)] & $1.14(0.36)$ & $1.21(0.35)$ & 1.281 & 0.893 & $1.02(0.29)$ & $1.39(0.35)$ & 8.16 & 0.294 \\
\hline LDL-C [mean (SD)] & $2.87(0.87)$ & $2.42(0.93)$ & -3.803 & 0.774 & $2.93(0.97)$ & $2.64(0.93)$ & -2.206 & 0.636 \\
\hline
\end{tabular}

Among the traditional risk factors of Han subjects, the prevalence of hypertension, fasting blood glucose levels, and TG levels differed significantly between the two groups (all $\mathrm{P}<0.05$ ). There was no significant difference in the following variables between $\mathrm{AF}$ patients and control subjects in the Han population: body mass index (BMI), alcohol consumption, systolic blood pressure, diastolic blood pressure, TC, HDL-C, LDL-C, age, and gender (all $\mathrm{P}>0.05$ ). In Kazak subjects, the following variables differed significantly between the two groups: fasting blood glucose, alcohol consumption, TG, and diastolic blood pressure (all $\mathrm{P}<$ 0.05). There was no significant difference in the following variables between Kazak AF patients and control subjects: hypertension, BMI, systolic blood pressure, TC, HDL-C, LDL-C, age, and gender (all $\mathrm{P}>0.05$ ).

\section{Distribution of the TaqI B polymorphism in AF patients and controls}

The genotype distribution of this polymorphism did not show a significant difference from Hardy-Weinberg equilibrium in either ethnic group ( $\mathrm{P}>0.05$ in the AF group and the control group). The frequency of the wild-type TaqI B genotype of CETP was significantly 
higher in AF patients than in control subjects in both ethnic groups (Table 2). The frequency of the $\mathrm{C}$ allele in $\mathrm{AF}$ patients was higher than in control subjects in both the Han and Kazak populations (Table 2). The carriers of the B1B1 genotype were more frequent among AF patients than among controls in both the Han population $\left(34.7 v s 26.4 \% ; \chi^{2}=10.686, \mathrm{P}=0.001\right)$ and the Kazak population $\left(53.4\right.$ vs $\left.24.8 \% ; \chi^{2}=27.802, \mathrm{P}<0.001\right)$. The odds ratio (OR) for carriers of the B1B1 genotype for AF was 0.187 (95\% confidence interval $(\mathrm{CI})=0.071-0.491)$ in the Han group and $8.426(95 \% \mathrm{CI}=2.295-30.933)$ in the Kazak population. After adjustment of confounding factors such as gender, age, smoking, alcohol consumption, hypertension, diabetes, smoking, systolic blood pressure, diastolic blood pressure, and the serum concentrations of HDL-C and LDL-C, the difference remained significant in the Han group $(\mathrm{P}=0.001$; OR $=0.187,95 \% \mathrm{CI}=0.071-0.491)$ and in the Kazak group $(\mathrm{P}=0.001 ; \mathrm{OR}=8.426,95 \% \mathrm{CI}=$ 2.295-30.933) (Table 3).

\begin{tabular}{|c|c|c|c|c|c|c|c|c|c|c|}
\hline & Genotype & $\mathrm{AF}[\mathrm{N}(\%)]$ & Control [N (\%)] & $\overline{\chi^{2}}$ & $\mathrm{P}$ value & Allele & $\mathrm{AF}$ (frequency) & Control (frequency) & $\overline{\chi^{2}}$ & P value \\
\hline \multirow[t]{4}{*}{ Han } & B2B2 & $10(0.099)$ & $38(0.295)$ & - & - & B1 & 0.624 & 0.485 & - & - \\
\hline & B1B1 & $35(0.347)$ & $34(0.264)$ & 10.686 & 0.001 & & & & & \\
\hline & B1B2 & $56(0.554)$ & $57(0.441)$ & 11.491 & 0.001 & B2 & 0.376 & 0.515 & 15.410 & 0.000 \\
\hline & $\mathrm{B} 1 \mathrm{~B} 2+\mathrm{B} 1 \mathrm{~B} 1$ & $91(0.901)$ & $91(0.705)$ & 13.119 & 0.000 & & & & & \\
\hline \multirow[t]{4}{*}{ Kazak } & B2B2 & $8(0.078)$ & $34(0.336)$ & - & - & B1 & 0.728 & 0.456 & - & - \\
\hline & B1B1 & $55(0.534)$ & $25(0.248)$ & 27.802 & 0.000 & & & & & \\
\hline & B1B2 & $40(0.388)$ & $42(0.416)$ & 9.920 & 0.002 & B2 & 0.272 & 0.544 & 10.090 & 0.001 \\
\hline & $\mathrm{B} 1 \mathrm{~B} 2+\mathrm{B} 1 \mathrm{~B} 1$ & $95(0.922)$ & $67(0.663)$ & 20.917 & 0.000 & & & & & \\
\hline
\end{tabular}

\begin{tabular}{|c|c|c|c|c|c|c|c|c|c|c|c|c|}
\hline & \multicolumn{6}{|c|}{ Kazak } & \multicolumn{6}{|c|}{ Han } \\
\hline & B & SE & Wald & $\mathrm{P}$ & OR & $95 \% \mathrm{CI}$ & $\mathrm{B}$ & SE & Wald & $\mathrm{P}$ & OR & $95 \% \mathrm{CI}$ \\
\hline B1B1 & 2.131 & 0.664 & 10.318 & 0.001 & 8.426 & $2.295-30.933$ & -1.677 & 0.492 & 11.603 & 0.001 & 0.187 & $0.071-0.491$ \\
\hline Hypertension & 1.971 & 0.571 & 11.902 & 0.001 & 7.181 & $2.343-22.011$ & -0.701 & 0.336 & 4.354 & 0.037 & 0.496 & $0.257-0.958$ \\
\hline DBP & 0.109 & 0.023 & 23.205 & 0.000 & 1.115 & $1.067-1.165$ & 0.024 & 0.013 & 3.579 & 0.059 & 1.024 & $0.999-1.050$ \\
\hline FBG & 1.280 & 0.413 & 9.586 & 0.002 & 3.597 & $1.600-8.090$ & -0.090 & 0.095 & 0.902 & 0.342 & 0.914 & $0.759-1.100$ \\
\hline HDL & 4.734 & 0.953 & 24.689 & 0.000 & 113.747 & $17.578-736.062$ & -1.018 & 0.512 & 3.958 & 0.047 & 0.361 & $0.133-0.985$ \\
\hline LDL & -1.104 & 0.344 & 10.286 & 0.001 & 0.331 & $0.169-0.651$ & 0.689 & 0.183 & 14.111 & 0.000 & 1.991 & $1.390-2.851$ \\
\hline Constant & -19.384 & 3.497 & 30.722 & 0.000 & 0.000 & & 1.161 & 1.894 & 0.376 & 0.540 & 3.193 & \\
\hline
\end{tabular}

For abbreviations, see legend to Table 1.

\section{DISCUSSION}

Xinjiang is a region comprising many ethnic groups, of which Kazak is the third largest group. We here found that the minor allele of the TaqI B polymorphism of the CETP gene had a higher frequency in AF patients than in controls in the Han and Kazak populations of Xinjiang.

The main physiological function of CETP is to transfer cellular lipid droplets and neutral lipids among cells, and further induces the selective uptake of fatty tissues of HDL-C and plays a role in the inflammatory response. Some studies have linked CETP polymorphisms to cardiovascular disease, hypertension, and even longevity (Thornton-Wells et al, 2004; Moore et al., 2006).

A Framingham nested study in a Caucasian population (Asselbergs et al., 2006) indicated a correlation between CETP polymorphisms and AF and a correlation between non- 
valvular AF and the TaqI B polymorphism of the CETP gene, which participates in the reverse transfer of cholesterol (Barter et al., 2004). In another study, Xu et al. (2008) found that the B1 allele of the CETP gene was an independent risk factor for genetic susceptibility of nonvalvular AF among the Han Chinese population of Zhejiang Province. Of all the candidate longevity genes identified to date, the apoliprotein E (APOE), CETP, and ACE genes have been correlated with cardiovascular diseases.

The TaqI B polymorphism of the CETP gene affects the HDL level to a large extent, and plays a genetic-environmental interactive role in the occurrence of cardiovascular diseases (Jensen et al., 2008). CETP polymorphisms result in different responses of individuals to statins, especially HDL-C. This is mainly because CETP polymorphisms are related to the effect of statin treatment lowering LDL-C levels (Anagnostopoulou et al., 2007).

The TaqI B polymorphism of the CETP gene is correlated with AF, and is particularly associated with the restraining responses of atrial fibroblasts to oxidation and inflammation, thus reducing vascular wall inflammation and restraining congestive heart failure that induces fibrosis. CETP gene variations may prevent the initiation of (preclinical) atherosclerosis and subsequently the development of AF (Korantzopoulos et al., 2003; Barzilai et al., 2003; Ostgren et al., 2004; Morita et al., 2006; Yang et al., 2008). However, the underlying biological mechanism explaining the association between AF and the TaqI B polymorphism of the CETB gene remains unclear.

In conclusion, in our sample of patients from Western China, AF was associated with the B1B1 genotype of CETP TaqI B. This result may broaden our knowledge of genetic variants and disease association studies. Genome-wide association studies are merited in different populations to further explore this relationship.

\section{Study limitation}

The study was limited by the relatively small sample size because of the relatively lower AF incidence in the Kazak population. This may have led to weak statistical significance in the analysis.

\section{ACKNOWLEDGMENTS}

2011).

Research supported by the Natural Science Foundation of China (NSFC; \#81060017-

\section{REFERENCES}

Anagnostopoulou K, Kolovou G, Kostakou P, Mihas C, et al. (2007). Pharmacogenetic study of cholesteryl ester transfer protein gene and simvastatin treatment in hypercholesterolaemic subjects. Expert. Opin. Pharmacother. 8: 2459-2463.

Asselbergs FW, Moore JH, van den Berg MP, Rimm EB, et al. (2006). A role for CETP TaqIB polymorphism in determining susceptibility to atrial fibrillation: a nested case control study. BMC Med. Genet. 7: 39.

Barter PJ, Nicholls S, Rye KA, Anantharamaiah GM, et al. (2004). Antiinflammatory properties of HDL. Circ. Res. 95: 764-772.

Barzilai N, Atzmon G, Schechter C, Schaefer EJ, et al. (2003). Unique lipoprotein phenotype and genotype associated with exceptional longevity. JAMA 290: 2030-2040.

Gauthier A, Lau P, Zha X, Milne R, et al. (2005). Cholesteryl ester transfer protein directly mediates selective uptake of high density lipoprotein cholesteryl esters by the liver. Arterioscler. Thromb. Vasc. Biol. 25: 2177-2184. 
Guidelines Subcommittee (1999). World Health Organization-International Society of Hypertension Guidelines for the Management of Hypertension. J. Hypertens. 17: 151-183.

Jensen MK, Mukamal KJ, Overvad K and Rimm EB (2008). Alcohol consumption, TaqIB polymorphism of cholesteryl ester transfer protein, high-density lipoprotein cholesterol, and risk of coronary heart disease in men and women. Eur. Heart J. 29: 104-112.

Korantzopoulos P, Kolettis T, Siogas K and Goudevenos J (2003). Atrial fibrillation and electrical remodeling: the potential role of inflammation and oxidative stress. Med. Sci. Monit. 9: RA225-RA229.

Li YY, Zhou CW, Xu J, Qian Y, et al. (2012). CYP11B2 T-344C gene polymorphism and atrial fibrillation: a metaanalysis of 2,758 subjects. PLoS One 7: e50910.

Liu Z, Zhou C, Liu Y, Wang S, et al. (2012). The expression levels of plasma micoRNAs in atrial fibrillation patients. PLoS One 7: e44906.

Lu WH, Mu HY, Liu ZQ, Yang YC, et al. (2012). The prevalence and distributing feature of atrial fibrillation in Xinjiang Uygur Autonomous Region Kazaks adult population. Zhonghua Nei Ke. Za Zhi 51: 674-676.

Moore JH, Gilbert JC, Tsai CT, Chiang FT, et al. (2006). A flexible computational framework for detecting, characterizing, and interpreting statistical patterns of epistasis in genetic studies of human disease susceptibility. J. Theor. Biol. 241: 252-261.

Morita A, Nakayama T, Doba N, Hinohara S, et al. (2006). Polymorphism of the C-reactive protein (CRP) gene is related to serum CRP level and arterial pulse wave velocity in healthy elderly Japanese. Hypertens. Res. 29: 323-331.

Mu HY, Qiu P, Lu WH, Liu ZQ, et al. (2010). Electrocardiogram Minnesota codings from 30,000 adult cases with Kazakh ethnicity in Xinjiang, China. Zhonghua Liu Xing. Bing. Xue Za Zhi 31: 451-454.

Nattel S (2002). New ideas about atrial fibrillation 50 years on. Nature 415: 219-226.

Ostgren CJ, Merlo J, Rastam L and Lindblad U (2004). Atrial fibrillation and its association with type 2 diabetes and hypertension in a Swedish community. Diabetes Obes. Metab. 6: 367-374.

Thornton-Wells TA, Moore JH and Haines JL (2004). Genetics, statistics and human disease: analytical retooling for complexity. Trends Genet. 20: 640-647.

World Health Organization Study Group (1985). Diabetes mellitus. WHO Tech. Rep. Ser. 727: 1-104.

Xiang X, Ma YT, Fu ZY, Yang YN, et al. (2009). Haplotype analysis of the CYP8A1 gene associated with myocardial infarction. Clin. Appl. Thromb. Hemost. 15: 574-580.

Xu LX, Yang WY and Zhang HQ (2008). Research on the relationship CETP TaqIB, KCNEl S38G and eNOS T-786C polymorphism and nonvalvular Atrial Fibrillation. Chin. J. Epi. Med. 5: 486-492.

Yang WY, Xu LX, Zhang HQ and Tao ZH (2008). Research on the relationship CETP and CRP polymorphism and atrial fibrillation of Han population. Chin. J. Heredity Med. 2: 225-229.

Zhou ZQ, Hu DY, Chen J, Zhang RH, et al. (2004). An epidemiological survey of atrial fibrillation in China. Zhonghua Nei Ke. Za Zhi 43: 491-494. 